\title{
The Cultural Value of Radio Astronomy
}

\author{
Woodruff T. Sullivan, III \\ Dept. of Astronomy Box 351580 Univ. of Washington \\ Seattle, WA 98195 USA \\ woody@astro.washington.edu
}

\begin{abstract}
In order to compete successfully in the marketplace of the radio spectrum radio astronomers must appeal not to economic gain, but to the cultural value of their enterprise. In the real world this can be problematic, but it is not hopeless. This paper gives arguments why radio astronomy, no less than astronomy as a whole, has great cultural value whether considered from an environmental or an intellectual point of view.
\end{abstract}

\section{Introduction}

Protecting and preserving radio astronomy takes place in the political arena, where the larger body politic, society as a whole, makes the vital decisions. The political process leading to these decisions considers economic, social and cultural values. Therefore, if we are to be effective in protecting radio astronomy, we must marshal strong and persuasive arguments that address as many of these factors as possible.

In this paper I argue that our strongest hand in demonstrating the worth of radio astronomy is in the cultural realm. The economic and social factors are, unfortunately, woefully and powerfully against us. When dealing with telecommunications firms, we bring only bad economic news to their managers, their satellite designers and their stockholders. What we are asking will cost them money and time and be a hindrance to providing the services eagerly sought by their public (and by many of us, too!). We must thus appeal to other countervailing values if radio astronomy signals are to survive amidst the cacophony of modern communications systems ${ }^{1}$.

There are two primary aspects of the cultural value of radio astronomy. The first is environmental and has the potential of becoming a powerful approach, but at this point requires more sensitization and education of the public and

\footnotetext{
${ }^{1}$ As discussed by Finley in this volume, the situation is vastly different with regard to light pollution. In addition to the cultural arguments about the value of (optical) astronomy, the International Dark-Sky Association and its allies have established that making the changes desired by astronomers also yields economic, social, aesthetic and environmental benefits to the general public - it is a win-win situation (see the article by Crawford in this volume). Progress is happening and at an accelerating pace, although there remains the need for a huge educational and political effort, and at optical wavelengths there are no large regulatory bodies with which to work.
} 
of decision-makers. The second is intellectual and is our ultimate strength. If we cannot persuade people that we are asking profound questions and finding exciting and important answers, then the battle is lost.

\section{Radio Astronomy as an Environmental Issue}

The history of the past forty years has seen a steady enlargement in what are considered "environmental" issues. Initially attention was focused on the more obvious dangers to the fundamental natural resources of air, water, soil, and flora and fauna. Environmentalists today, however, realize that other, more subtle, entities also affect the well-being of planet Earth and its inhabitants. Examples include the stratospheric layers (ozone depletion), general burning of hydrocarbons (global warming), the sonic environment (noise pollution) and the night-time environment (light pollution). For its environmental welfare the public is now accustomed to considering new phenomena and new ways of looking at old situations.

So it is with radio astronomy, an abstract and arcane field of whose value we must persuade the average person. Radio astronomers must start talking about the electromagnetic spectrum as a finite resource, not unlike virgin forests. Just as with the forests, the great bulk of the spectrum resource has gone to serve the material, everyday needs of society. The question is what to do with the remaining small portion? Adding it to the other "useful" spectrum will minimally enhance overall economic prosperity, but will leave us with zero for radio astronomy. Is the trade-off worth it? Once again, this is a political issue, but it is winnable - with proper education the public on the whole is supportive of preservationist stances.

The task then is to convince the public that the value of radio astronomy is not unlike the value of a forest of 500-year-old trees. In the case of the forest, our emotions respond to its magnificence and our intellect craves to study its unique and fragile ecosystem. In the case of radio astronomy, can we likewise show that fundamental human needs are satisfied? First, we must show the sustaining and exciting nature of astronomy as a whole, and then demonstrate that radio astronomy is one of astronomy's key components. People must value scientists probing the Universe to better determine what's there and how it works, searching for clues about where we fit in the Big Picture. Then they must be made aware of how our society is endangering our ability to view this Universe.

We need not start from scratch. In a recent article on the concept of the commons, environmentalists Snider and Warshall (1998) have discussed a wide variety of common resources, ranging from local fisheries to public roads to sacred heritages to humankind's legacy of ethics. And amongst their list of thirty entities is the electromagnetic spectrum, or "spectral commons"! Once this principle becomes established, namely that the electromagnetic spectrum should be treated as a public commons, then the way is greatly eased for preservation of a portion of it for other than practical uses. We can also appeal to the familiar warning of the "tragedy of the commons". Ecologist Garrett Hardin coined this phrase for the erosion and eventual destruction of any commons that can occur 
if each person acts only in his or her own self-interest, without regard to the interests of the community.

Morimoto (1993) has called the beautiful view of the Universe afforded by a dark sky, whether optical or radio, a "rare and precious treasure of Nature". Paul Vanden Bout (1994) has nicely developed the environmental analogy in his article "Preserving wilderness areas in the radio spectrum". He points out that $1 \mathrm{MHz}$ of bandwidth in the cellular telephone band may have a value of one billion dollars, but does that mean it should all be sold to the highest bidder? What is the value of the acreage of the Grand Canyon, or of the Great Barrier Reef, or of Fujiyama? Vanden Bout points out that spectrum is like public lands:

It is fixed in overall amount, controlled by the government, serving many varied constituencies, with important roles in the economy and safety of the nation. In this view of the spectrum, to give a few examples, the protected radio astronomy bands are analogous to wilderness areas and national parks, the navigation bands to ... defense installations and the broadcast bands ... to lands leased for cattle grazing or mineral extraction. Market forces are applied after recognizing the importance of some services beyond their purely economic value.

Continuing the analogy, he points out that the lack of wisdom in siting heavy industrial activities adjacent to a national park is the same as that of assigning frequencies for ubiquitous and powerful satellite transmitters next to those for radio astronomy.

Reserving slices of spectrum as wilderness is of course an abstract concept. But we can also more directly profit from the concept of wilderness areas by seeking actual zones or regions on the Earth free of humanmade interference. The largest and earliest such zone is the National Radio Quiet Zone, a region of $\sim 160 \mathrm{~km} \times 160 \mathrm{~km}$ established in 1956 for the benefit of the National Radio Astronomy Observatory at Green Bank, West Virginia, USA. At this symposium Butcher has argued for the creation of one or more International Radio Quiet Zones to protect the next generation of radio telescopes, such as the Square Kilometre Array. The ultimate such zone would be the far side of the Moon and indeed some are working to that end, but this is only a partial (and very expensive) solution and only for the distant future. Radio astronomy from the Moon, if and when it happens, will no more eliminate the need for groundbased radio observatories than the orbiting Hubble Space Telescope has made ground-based optical observatories unnecessary.

\section{Radio Astronomy as an Intellectual Value}

To make our case one must first establish the intellectual value of all of astronomy, then focus on radio astronomy and show that it is integral to modern astronomy. The cultural value and impact of astronomy as a whole, however, is a huge topic and beyond the scope of this paper. Plato even said in the Timaeus (47a) that philosophy itself began with astronomy: 
For had we never seen the stars and the sun and the heaven, none of the words which we have spoken about the universe would ever have been uttered ... [These] have created number and have given us a conception of time and the power of inquiring about the nature of the universe. And from this source we have derived philosophy.

If one were to write a book on "astronomy and culture", some of the possible chapter titles reveal how intimately astronomy is woven into who we are and how we see ourselves: time and calendrics, navigation, astrology, eclipses, art and music, extraterrestrial life, physics, etc. It might be thought that most of these connections apply only to the past, but this is wrong on almost every count. A few examples: (1) regarding time and navigation, the Global Positioning Satellite (GPS) system (which was largely designed by astronomers and is still fine-tuned by the US Naval Observatory) is transforming not just navigation, but everything from ornithology to police work; (2) regarding the arts, cosmic themes are still frequent in modern art (and certainly in popular culture); and (3) regarding astrology and extraterrestrial life, widespread beliefs not unlike those of the past are rampant (and astronomy needs to exist to weigh in with its scientific input); and (4) regarding new physics, the "astronomical laboratory" still supplies unmatched testing grounds for nuclear physics, high-energy physics and gravitation.

Astronomy as a whole thus continues as a vital part of culture, as it always has. But what about radio astronomy, which has been with us for only a half century? An examination of its short history reveals its critical role in our present understanding of the cosmos. Radio waves were the first non-optical portion of the spectrum to be exploited in a major way. The astronomy of the 1940 s and 1950s was revolutionized by discoveries of radio sources that allowed probing of the farthest reaches of the Universe, relativistic electrons threading the Milky Way, compact sources of unprecedented energy, dark cool clouds that traced our Galaxy's rotation, and huge bursts of energy in the solar corona. As radio astronomy matured in the 1960 s and 1970 s it led to the discovery of quasars, the cosmic microwave background radiation, pulsars and complex interstellar molecules. The last two decades have seen the first evidence for gravitational waves (emitted by a pulsar system), pulsar clocks as accurate as anything on Earth, excellent evidence for the presence of huge black holes at the centres of galaxies, and mapping of the early Universe's structure through subtle corrugations in the cosmic background.

\section{A Few Examples of Radio Astronomy's Importance}

Rather than try to cover all of the major contributions of radio astronomy, this section reviews three of the most important in more detail. These examples have been chosen because of the profound effect they have had on how we view our Universe. As it turns out, these have also led to no less than three Nobel Prizes.

\subsection{Cosmic Background Radiation}

Using a strange, sugar-scoop-shaped antenna at Bell Labs in New Jersey, Penzias and Wilson (1965) deployed a sensitive and stable receiver system for a study 
of the Milky Way's background synchrotron radiation. The experiment was exquisitely designed and allowed an absolute measurement of radio intensity from any direction in the $\mathrm{sky}^{2}{ }^{3}$.

The surprising finding was that there was in fact no sector of the sky that was radio-quiet: rather, the entire sky emitted as if at a uniform temperature of $3 \mathrm{~K}$. This radiation soon came to be accepted in the context of the Big Bang theory as the cooled, redshifted remnant of an originally hot, dense Universe. Three decades of subsequent measurements, most accurately by the COBE satellite in the mid 1990s, have confirmed this basic idea to the point where this cosmic background radiation is still looked upon as one of the two or three key pieces of observational evidence underpinning Big Bang cosmology.

\subsection{Pulsars}

Once again, a serendipitous observation, doggedly tracked down, led to a basic discovery. This time it was an array of 2,048 dipole antennas at Cambridge University in England, designed to study fast intensity scintillations that radio sources exhibit because of variable propagation conditions (primarily changing electron density in the interplanetary medium). But amongst the expected scintillating sources were discovered four that changed not in the usual irregular fashion, but with incredible regularity. Already in their first article Hewish, Bell, Pilkington, Scott and Collins (1968) mentioned the possibility that these pulsating sources might be neutron stars, which is still the best interpretation. Neutron stars had been talked about as a theoretical construct since the 1930s, but here was the first observational evidence for their existence. They are the fast-rotating, collapsed-core remnants of supernova explosions, superdense objects with the mass of a star but the size of a mountain. Besides providing a powerful laboratory for testing ideas of the physics of matter under such extreme conditions, the pulsars (of which hundreds are now known) are beautiful clocks. The most accurate are those with the fastest periods (approaching one millisecond) and their long-term stability is as good as any of the manmade time standards on Earth.

One pulsar was found by Hulse and Taylor (1975) to be in orbit about an unseen companion object (itself soon inferred to be a neutron star, but radio quiet). A monitoring program using the $305-\mathrm{m}$ diameter Arecibo dish soon revealed that the regular orbital motion of the pulsar about its companion every 7.75 hours was slowly shifting in a complex manner. The stability of the pulsar's 59.03 msec periodicity (about 1 part in $10^{17}$ ) and the accumulation of many measurements of it, each accurate to 14 decimal places, allowed the subtle orbital changes to be detected and ascribed to a variety of relativistic effects. While most of these effects had been measured in other contexts, one provided wholly new evidence for Einstein's general theory of relativity, which predicts that oscillating masses should emit gravitational waves roughly analogous to

\footnotetext{
${ }^{2}$ In contrast, almost all radio astronomical measurements, then as now, are not absolute, but relative to a nearby patch of sky assumed to have no signal.

${ }^{3}$ It is ironic that this fundamental discovery was made with technology available as an offshoot of the then nascent development at Bell Labs of the first earth-orbiting communication satellites (Telstar, Early Bird, etc.).
} 


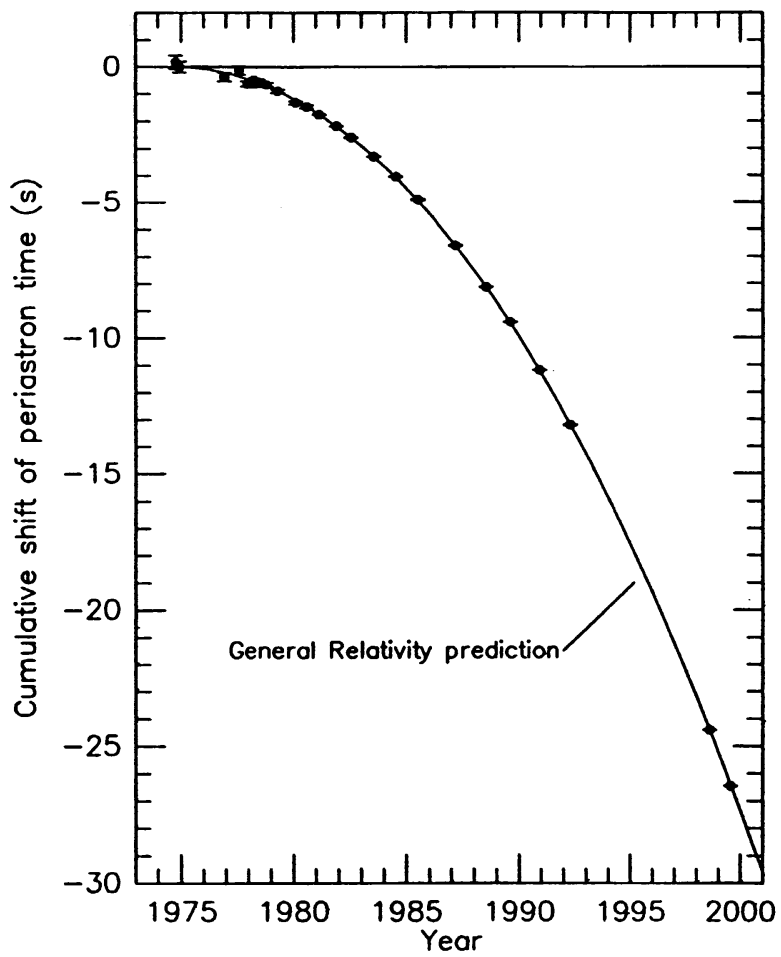

Figure 1. Cumulative effect of the orbital decay of the binary pulsar PSR B1913+16 over the past 25 years. The points are measured values based on timing of the pulsar at Arecibo Observatory, while the line is the effect expected from general relativistic emission of gravitational waves. (Courtesy J.H. Taylor)

how oscillating electrons emit electromagnetic waves. Despite many previous attempts to detect such waves directly, no one had succeeded. Within a decade of monitoring, however, Taylor and his colleagues showed that the pulsar's orbit was decaying exactly as would be expected if the two, orbiting neutron stars were losing energy via emission of gravitational waves. The radio technique's marvellous sensitivity uncovers the tiny decrease in every orbital period, two parts in $10^{12}$, or $76 \mu$ sec per year (Fig. 1). Our best theory of gravity became even better.

\subsection{Microwave and Millimetre Spectroscopy of Molecules}

Astronomical spectroscopy is an essential technique. By measuring radiation at distinctive frequencies (spectral lines) characteristic of each element and molecule, radio astronomers can determine not only the density and location of these species, but also their velocity, temperature, pressure, ambient magnetic field, etc. The first radio spectral line to be detected (in 1951) and still 

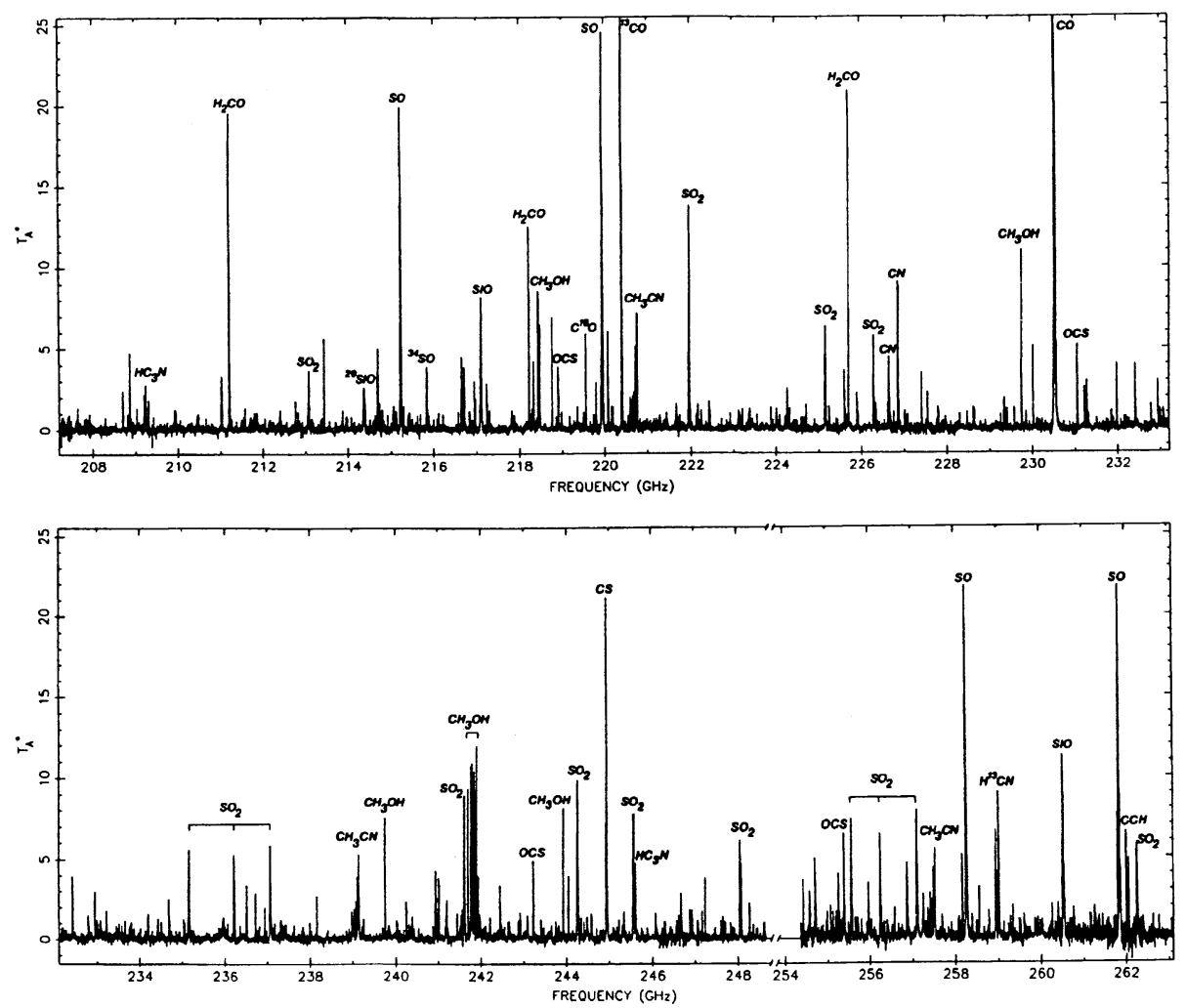

Figure 2. The spectrum of the Orion Molecular Cloud over the range 208 to $262 \mathrm{GHz}$, as measured at the Owens Valley Radio Observatory. Molecular identifications are given for the strongest of more than 800 spectral lines detected in this range. (Adapted from Blake et al. 1987)

the most important, was that at $1.420 \mathrm{GHz}$ from the hydrogen atom, the most abundant element in the Universe. But the real floodgates opened in the late 1960 s when a varied suite of molecules was found to be present in many interstellar regions of our galaxy the Milky Way. First spectral lines to be found were hydroxyl $(\mathrm{OH})$, water vapor $\left(\mathrm{H}_{2} \mathrm{O}\right)$, ammonia (NH3), carbon monoxide $(\mathrm{CO})$, cyanide $(\mathrm{CN})$, hydrogen cyanide $(\mathrm{HCN})$ and formaldehyde $\left(\mathrm{H}_{2} \mathrm{CO}\right)$. By now over 140 different molecular species, some with up to 13 atoms, have been detected. They include ethyl alcohol (!, $\left.\mathrm{CH}_{3} \mathrm{CH}_{2} \mathrm{OH}\right)$, the sugar glycoaldehyde $\left(\mathrm{C}_{2} \mathrm{H}_{4} \mathrm{O}_{2}\right)$ and perhaps even the amino acid glycine $\left(\mathrm{H}_{2} \mathrm{NCH}_{2} \mathrm{COOH}\right)$. They reveal a previously unsuspected, rich carbon-based chemistry not unlike what may have occurred in the early solar system long before life appeared on Earth. Through the study of these molecules our ideas about the formation of stars and planets have been revolutionized.

With few exceptions these spectral lines happen to occur at frequencies above $20 \mathrm{GHz}$, most above $100 \mathrm{GHz}$. Figure 2 shows a spectrum over a range 
from 208 to $262 \mathrm{GHz}$, with some of the rich array of spectral lines identified. These all originate from a single direction in the sky, a particularly dense and dusty interstellar cloud and region of star formation.

\section{Conclusion}

Whether considered from an environmental or a cultural point of view, radio astronomy is a resource vital to society. It is the duty of astronomers to demonstrate this to the public at large and to those who make decisions regarding usage of the radio spectrum. How foolish it would be if, after half a century of exciting radio discoveries that have profoundly affected how we view our Universe, society were to squelch this activity by wrapping the Earth in an impenetrable electromagnetic fog.

\section{References}

Blake G. A., Sutton E. C., Masson C. R. \& Phillips T. G. 1987, Ap. J. 315, 621 Hewish, A., Bell, S.J., Pilkington, J.D.H., Scott, P.F.\& Collins, R.A. 1968, Nature 217,709.

Hulse, R. \& Taylor, J.H. 1975, Ap. J. L. 195, L51.

Morimoto. M. 1993, Modern Radio Science 1993, 213.

Penzias, A.A. \& Wilson, R.W. 1965, Ap J 142, 419.

Snider, G. \& Warshall, P. 1998, Whole Earth, Fall issue, 4-7 + 16-23 + 50.

Vanden Bout, P.A. 1994, in The Vanishing Universe, Ed. D. McNally (Cambridge: Cambridge Univ. Press), p. 97. 